\title{
Review On Google Android a Mobile Platform
}

\author{
Shyam Bhati ${ }^{1}$, Sandeep Sharma ${ }^{2,}$ Karan Singh ${ }^{3}$ \\ ${ }^{1,3}$ (Department of Computer Science and Engineering, SOICT Gautam Buddha University, India) \\ ${ }^{2}$ (Department of Electronics and Communication Engineering, SOICT Gautama Buddha University, India)
}

\begin{abstract}
At present smart phone usage is increasing dramatically. Globally, if a usage comparison can be carried out between cell phone and smart phone devices. Smart phone is a personnel device which provides entertainment, information, making call and writing SMS. Customization makes cell phone a smart phone (installation and uninstallation of application). The increasing importance of smart phone devices has triggered intense competition among technology giants to capture the bigger market share for mobile platform. So Google launched Android, an open source mobile platform for smart phone devices consisting of a Linux Kernel, runtime environment, development framework, libraries and key applications. Since its official public release, Android has captured the market. This paper intends to explore conventional approach of mobile application execution, an approach of mobile application execution in Android \& comparison between Android OS (Google), iOS (Apple) \& Blackberry OS (RIM).

Keywords - Android, iOS, Blackberry, Open source mobile platform.
\end{abstract}

\section{INTRODUCTION}

Android is an open source mobile platform provided by Google. This is a software stack for mobile devices which includes an operating system, applications development framework, runtime environment and library. Android's mobile operating system is based on a modified version of the Linux kernel [1]. The application development uses Java programming language and the virtual mechine that optimizes the usage of memory and resources [2]. Since its official public release, Android has captured the interest from the market. The Android Market [7] has experienced tremendous growth since its inception and has democratized application development by allowing anyone to upload applications to the market [3]. Google entered into the mobile market not as a handset manufacturer, but by launching mobile platform called as "Android" for mobile devices such as Smart phones and PDA in November 2007. Google has a vision that Android based cell phone will have all the functions available in the latest $\mathrm{pc}^{\mathrm{s}}$. In order to make this effort possible, Google launched the Open Handset Alliance. Google introduced Android as an OS which runs the powerful applications and gives the users a choice to select their applications and their carriers. Android is gaining strength both in the mobile industry and in other industries with different hardware architectures. The increasing interest from the industry arises from two core aspects: its open-source nature and its architectural model. Being an open source project, Android allows us to fully analyze and understand it, which enables feature comprehension, bug fixing, further improvements regarding new functionalities and finally, porting to new hardware. On the other hand, its Linux kernel-based architecture model also adds the use of Linux to the mobile industry, allowing taking advantage of the knowledge and features offered by Linux. The Android platform consists of several layers which provide a complete software stack. Android applications are Java-based and this factor entails the use of a virtual machine VM environment, with its advantages. Android uses its own VM called Dalvik, which interprets and executes portable Java-style byte code after transforming it, which is optimized to operate on the mobile platform. All of these aspects make Android an appealing target to be used in other type of environments [4].

The remainder of this paper is organized as follows: Section II briefly describes the Android mobile platform. Section III presents Android application execution process. Section IV briefly describes the Android market share. Finally Section V concludes this paper.

\section{ANDROID MOBILE PlatForm}

Android is a software stack provided by Google which provides the facility to develop, to deploy and to execute an application. Android platform is divided into 5 Layers [1][6]. Android architecture is shown in figure 1.

\section{A. Application}

A set of native applications are on the top level in the framework, including an email client, a SMS app, a calendar, a maps-application, web browser, contacts-app, and many more. All apps are written using the Java programming language. 


\section{B. Application Framework}

Application Framework is the base of developing Applications in Android, most time the developers are working with Application Framework. The main components of Application Framework are: the activity manager, the window manager, content providers, the view system, the notification manager, the package manager, the telephony manager, the resource manager, the location manager.

\section{Libraries}

Android includes a set of $\mathrm{C} / \mathrm{C}++$ libraries used by various components of the Android system. The main core libraries are: Media Framework, Web Kit, SGL, OpenGL ES, Free Type, SQLite, etc. These capabilities are exposed to developers through the Android application framework.

\section{Android Runtime}

Every Android application runs in its own process, with its own instance of the Dalvik virtual machine. Dalvik has been written so that a device can run multiple VMs efficiently.

\section{E. Linux Kernel}

Android relies on Linux (Kernel version 2.6) for core system services such as memory management, process management, network stack, security, and driver model. The core also acts as a hardware abstraction layer between the applications and all the hardware.

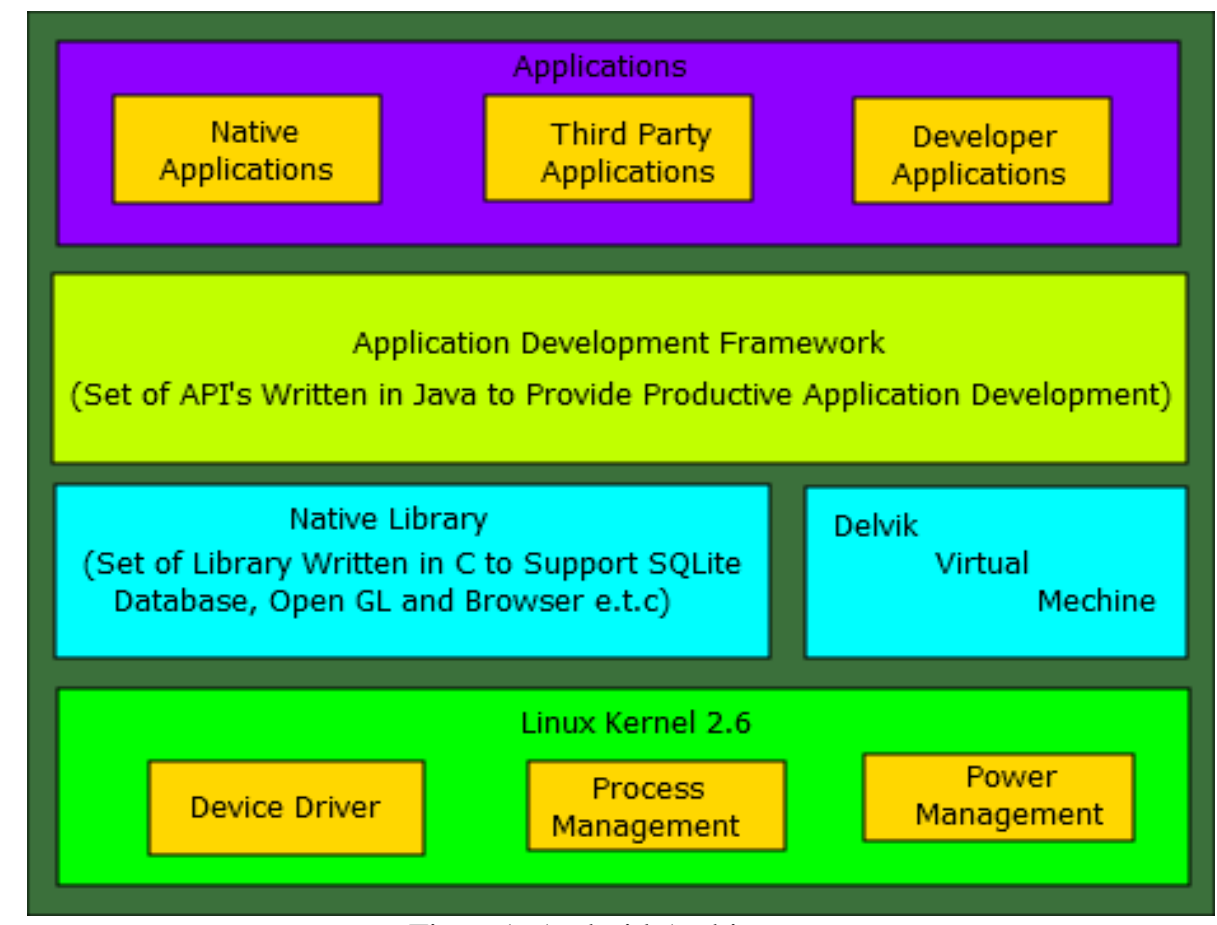

Figure1. Android Architecture

There are four types of components in Android. If a developer wants to use these components, the developer has to declare it in a Manifest file.

\section{A. Activity}

An activity represents a single screen with a user interface [5]. Activity is launched by the Intent.

\section{B. Service}

Service is a background process invisible to the user. For example, Service may perform the operations to play the music and download the file as a background process.

\section{Broadcast Receiver}

Broadcast Receiver is a component to receive the Broadcast Intent from the OS. For example, if the application wants to know the OS boot event, it has to receive the Broadcast Intent by using the Broadcast Receiver. 


\section{Content Provider}

Content Provider is a database. For example, the contact list and phone $\log$ are managed by this component.

\section{ANDROID APPLICATION EXECUTION PRECESS}

In this section two approaches are being discussed, first is conventional approach of mobile application execution and second is given by android is called android application mobile execution approach.

\section{A. Conventional approach of mobile application execution}

Conventional approach shown in figure 2 of J2ME application in any mobile was not so good. There were some problems in this approach that made it obsolete form the market.

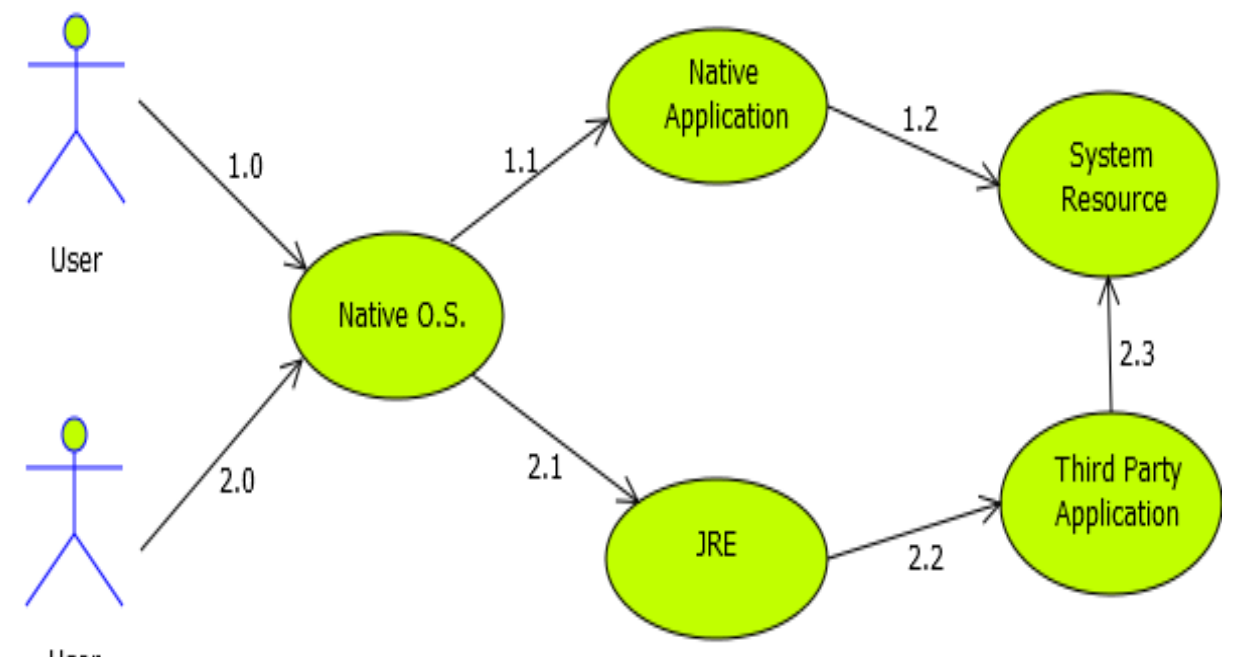

User

Figure 2. Conventional approach of mobile application execution

1.0 User requests to start native application.

1.1 Native OS starts native application as a process.

1.2 Native application has complete access to the system resource.

2.0 User requests to start third party application which is developed in J2ME.

2.1 Native OS starts JRE as a process.

2.2 JRE launches third party application.

2.3 Third party application has limited access to the system resource.

Some of limitations are being discussed here.

1) Third party applications were treated as second class citizen. That is all the system resources were not available to them and in case of processor or memory requirement third party applications were killed first.

2) If someone wants to develop an application in native technology, application developer must learn native APIs and hardware specification which were not available to them at large. For the reason of meaningful application, application developer require native library which is vendor specific.

Table I. Historical Sales of Smart Phone in Millions

\begin{tabular}{|l|l|l|l|l|l|l|}
\hline Year & Android & Blackberry & iOS & Symbian & $\begin{array}{l}\text { Windows } \\
\text { Mobile/Phone }\end{array}$ & Bada \\
\hline 2007 & $*$ & 11.77 & 3.3 & 77.68 & 14.7 & $*$ \\
\hline 2008 & $*$ & 23.15 & 11.42 & 72.93 & 16.5 & $*$ \\
\hline 2009 & 6.8 & 34.35 & 24.89 & 80.88 & 15.03 & $*$ \\
\hline 2010 & 67.22 & 47.45 & 46.6 & 111.58 & 12.38 & $*$ \\
\hline 2011 & 219.52 & 51.54 & 89.26 & 93.41 & 8.77 & $*$ \\
\hline $2012 \mathrm{Q} 1$ & 81.07 & 9.94 & 33.12 & 12.47 & 2.71 & 3.84 \\
\hline $2012 \mathrm{Q} 2$ & 104.8 & 7.4 & 26.0 & 6.8 & 5.4 & $*$ \\
\hline $2012 \mathrm{Q} 3$ & 122.5 & 9.0 & 23.6 & 4.4 & 4.1 & 5.1 \\
\hline $2012 \mathrm{Q} 4$ & 144.7 & 7.3 & 43.5 & 2.6 & 6.2 & 2.7 \\
\hline
\end{tabular}




\section{B. Android mobile application execution approach}

Flow of Android application execution is shown in figure 3. It completes in following steps:

1.0 User requests to start native application.

1.1 Kernel starts a process.

1.2 An instance of DVM is created.

1.3 DVM launches native application.

1.4 Native application has complete access to the system resource.

2.0 User requests to start third party application.

2.1 Kernel starts a process.

2.2 An instance of DVM is created.

2.3 DVM launches third party application.

2.4 Third party application has complete access to the system resource.

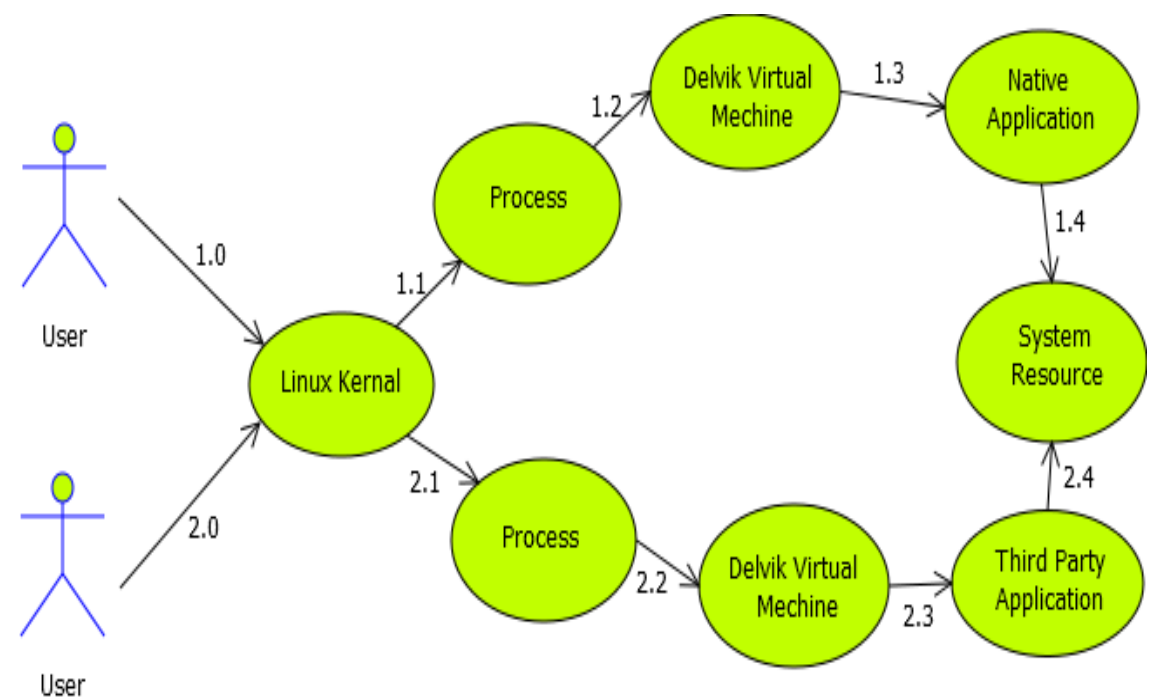

Figure 3. Android application execution approach

\section{ANDROID MARKET ANALYSIS}

The Android Market, an online software store, is developed by Google for Android devices. It was made available to users on October 22, 2008. Most of the Android devices come with preinstalled "Market" application which allows users to browse, buy, download, and rate different available applications and other content for mobile phones equipped with the open-source operating system [4].

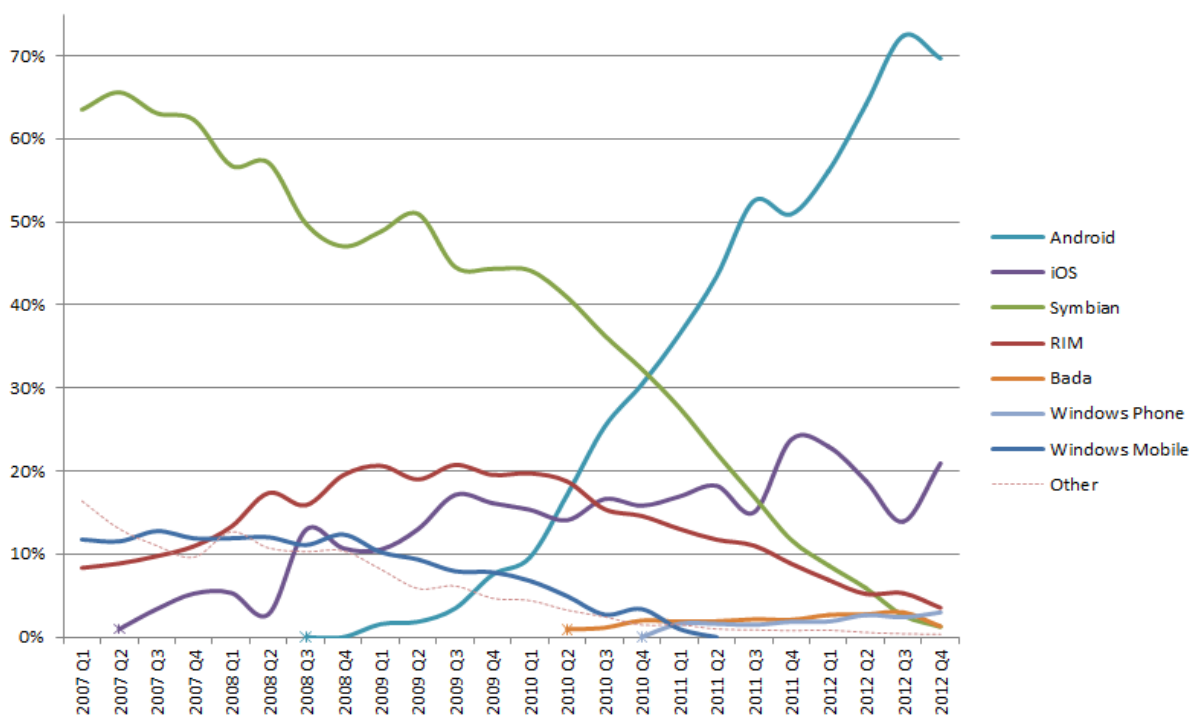

Figure 4. World-Wide Smartphone Sales (\%) 
Android apps may be obtained from any source including a developer's own website. Also, Android developers can create their own application market. Google does not have a strict requirement for the application to show up on the Android Market compared to the process used by Apple. Lastly, the Android Market follows a 70/30 revenue-sharing model for applications developed by developers. The developers of priced applications receive $70 \%$ of the application price and remaining 30\% distributes [4]. The global smart phone sell from 2007Q1 to 2012Q4 is shown bellow in figure 4. Some historical sale of smart phones in millions is shown in table I. This table shows how Android has captured the market interest. These records are from 2007 till 2012Q4. Android mobile phones is increasing in the market exponentially.

\section{CONCLUSION}

In this survey we have presented some details of Android, \& studied features as well as suitability of android for mobile devices. We have also compared Android with Symbian, Windows Mobile, Windows Phone, Bada, RIM and iOS. Findings of this survey are that Android is superior compare to its competitive operating systems. It acts as an Emerging Software Platform for Mobile Devices. Android is Open source mobile platform. It is key feature of Android that will make it a leader in mobile Platform.

\section{REFERENCES}

[1] Ki-Cheol Son, Prof. Jong-Y eol Lee," The Method of Android Application Speed up by using NDK"

[2] Robi Grgurina, Goran Brestovac, Tihana Galinac Grbac,'Development Environment for Android Application Development: an Experience Report", University of Rijeka, MIPRO 2011, May 23-27, Opatija, Croatia

[3] Ryan Johnson, Zhaohui Wang, Corey Gagnon, Angelos Stavrou," Analysis of Android Applications' Permissions", Sixth International Conference on Software Security and Reliability Companion, IEEE, 2012

[4] Nisarg Gandhewar, Rahila Sheikh," Google Android: An Emerging Software Platform For Mobile Devices", Nisarg Gandhewar et al. / International Journal on Computer Science and Engineering (IJCSE), ISSN : 0975-3397, 2010

[5] Jianye Liu, Jiankun Yu, "Research on Development of Android Applications", Fourth International Conference on Intelligent Networks and Intelligent Systems, IEEE, 2011

[6] Xiangling Fu, Xiangxiang Wu, Maoqiang Song, Mian Chen,” Research on Audio/Video Codec Based on Android”, IEEE, 2010

[7] Android Market. https://market.android.com 